\title{
GENERALISED EULER CONSTANTS
}

\author{
by J. KNOPFMACHER
}

(Received 28th July 1976)

\section{Introduction}

Let the Laurent expansion of the Riemann zeta function $\zeta(s)$ about $s=1$ be written in the form

$$
\zeta(s)=\frac{1}{s-1}+\sum_{i=0}^{\infty} \frac{(-1)^{i}}{i !} \gamma_{i}(s-1)^{i}
$$

It has been discovered independently by many authors that, in terms of this notation, the coefficient

$$
\gamma_{i}=\lim _{N \rightarrow \infty}\left\{\sum_{n=1}^{N} \frac{\log ^{i} n}{n}-\frac{\log ^{i+1} N}{i+1}\right\}
$$

in particular, $\gamma_{0}$ is the well known Euler constant $\gamma=0.57721$.. (see for example Briggs and Chowla (6), or van Veen (15)).

A generalisation of this conclusion covering ordinary Dirichlet series $f(s)=$ $\sum_{n=1}^{\infty} h(n) n^{-s}$ subject to a certain condition has been obtained by Briggs and Buschman (5), and Berndt (2) derived a similar result for the Hurwitz zeta function $\zeta(s, a)$, $0<a \leqslant 1$. The theorem of Briggs and Buschman applies to various special series of interest besides the particular examples listed by them. For example, by invoking a theorem of Weber, one sees that it applies to the Dedekind zeta function of an algebraic number field; this is discussed further below. Unfortunately, though, the theorem of Briggs and Buschman does not cover Berndt's theorem about the Hurwitz zeta function, and in general it is not applicable to the zeta functions of quadratic forms, or of generalised prime number systems, or of arithmetical semigroups and formations (as discussed respectively by Siegel (14), Bateman and Diamond (1), and the present author $(8,9)$, for example).

The first purpose of this note is to prove a theorem about certain Laplace transforms, which does cover all the above cases, and in addition gives error estimates for the limit relations of Briggs and Buschman, and Berndt. Then there is a discussion of various further applications to special functions of interest. Lastly, there are some remarks on a few special properties of the generalised Euler constants $\gamma_{i}(r, k)$ corresponding to the arithmetical progression of positive integers $r, r+k$, $r+2 k, \ldots(r \leqslant k)$; the particular constant $y_{0}(r, k)$ was studied previously by Briggs (4), and Lehmer (13). 


\section{Laurent expansions of Laplace transforms}

Although our present interest lies mainly with Corollary 1.2 below, we begin with more general result:

Theorem 1.1. Let $f(s)=\int_{0}^{\infty} e^{-s t} d F(t)$, where $F(t)$ is a real-valued function $c$ bounded variation in every finite interval $0 \leqslant t \leqslant y$, with the property that there exi: constants $C \neq 0$ and $\alpha, \beta$ with $0 \leqslant \beta<\alpha$ such that

$$
F(t)=C e^{\alpha t}+O\left(e^{\beta t}\right) \text { as } t \rightarrow \infty .
$$

Then the abscissa of convergence of $f(s)$ is $\alpha$, and $f(s)$ can be extended to an analyti function of $s$ for all complex $s$ with $\operatorname{Re} s>\beta, s \neq \alpha$, in such a way that, about $s=c$ $f(s)$ has a Laurent expansion of the form

$$
f(s)=\frac{\alpha C}{s-\alpha}+\sum_{i=0}^{\infty} \frac{(-1)^{i}}{i !} \gamma_{i}(F)(s-\alpha)^{i},
$$

where

$$
\gamma_{i}(F)= \begin{cases}\int_{0}^{y} e^{-\alpha t} d F(t)-\alpha C y+F(0)+O\left(e^{(\beta-\alpha) y}\right) & \text { if } i=0, \\ \int_{0}^{y} t^{i} e^{-\alpha t} d F(t)-\frac{\alpha C}{i+1} y^{i+1}+O\left(y^{i} e^{(\beta-\alpha) y}\right) & \text { if } i \geqslant 1 .\end{cases}
$$

Proof. The asymptotic hypothesis on $F(t)$ implies that

$$
\limsup _{t \rightarrow \infty} \frac{\log |F(t)|}{t}=\alpha,
$$

and so a standard theorem (Widder (16), page 42) implies that $f(s)$ has abscissa $c$ convergence $\alpha$. Thus $f(s)$ is analytic for $\operatorname{Re} s>\alpha$, and in this region $f(s)$ : $-F(0)+s \int_{0}^{\infty} e^{-s t} F(t) d t$.

Now let $R(t)=F(t)-C e^{\alpha t}$. Then, for $\operatorname{Re} s>\alpha$,

$$
f(s)=\frac{\alpha C}{s-\alpha}+C+s \int_{0}^{\infty} e^{-s t} R(t) d t .
$$

Since $R(t)=O\left(e^{\beta t}\right)$, a convenient analytical lemma stated by Bateman and Diamon (1), page 205, shows easily that $g(s)=s \int_{0}^{\infty} e^{-s t} R(t) d t$ is an analytic function of $s$ fo $\operatorname{Re} s>\beta$. Thus $f(s)$ can be extended as stated, and about $s=\alpha$ it has a Laurer expansion of the form

$$
f(s)=\frac{\alpha C}{s-\alpha}+\sum_{i=0}^{\infty} \frac{(-1)^{i}}{i !} \gamma_{i}(F)(s-\alpha)^{i},
$$

where $\gamma_{0}(F)=C+g(\alpha)$ and $\gamma_{i}(F)=(-1)^{i} g^{(i)}(\alpha)$ for $i \geqslant 1$.

Now, integration by parts and the asymptotic hypothesis on $F(t)$ gives

$$
\int_{0}^{y} e^{-\alpha t} d F(t)=F(y) e^{-\alpha y}-F(0)+\alpha C y+\alpha \int_{0}^{y} e^{-\alpha t} R(t) d t
$$




$$
\begin{aligned}
& =C+O\left(e^{(\beta-\alpha) y}\right)-F(0)+\alpha C y+g(\alpha)-\alpha \int_{y}^{\infty} e^{-\alpha t} R(t) d t \\
& =\alpha C y+\gamma_{0}(F)-F(0)+O\left(e^{(\beta-\alpha) y}\right) .
\end{aligned}
$$

Similarly, for $i \geqslant 1$,

$$
\begin{aligned}
\int_{0}^{y} t^{i} e^{-\alpha t} d F(t)= & F(y) y^{i} e^{-\alpha y}-\int_{0}^{y} F(t) d\left(t^{i} e^{-\alpha t}\right) \\
= & \frac{\alpha C}{i+1} y^{i+1}+O\left(y^{i} e^{(\beta-\alpha) y}\right)-\int_{0}^{\infty} R(t) d\left(t^{i} e^{-\alpha t}\right) \\
& +O\left(\int_{y}^{\infty} e^{\beta t} d\left(t^{i} e^{-\alpha t}\right)\right) \\
= & \frac{\alpha C}{i+1} y^{i+1}+\int_{0}^{\infty} t^{i} e^{-\alpha t} d R(t)+O\left(y^{i} e^{(\beta-\alpha) y}\right)
\end{aligned}
$$

But, for $\operatorname{Re} s>\beta$ and $i \geqslant 0$,

Therefore

$$
g^{(i)}(s)=(-1)^{i} \int_{0}^{\infty} t^{i} e^{-s t} d R(t) .
$$

$$
\int_{0}^{y} t^{i} e^{-\alpha t} d F(t)=\frac{\alpha C}{i+1} y^{i+1}+(-1)^{i} g^{(i)}(\alpha)+O\left(y^{i} e^{(\beta-\alpha) y}\right),
$$

and so the theorem follows.

Corollary 1.2. Let $\zeta_{G}(s)=\sum_{n=1}^{\infty} G\left(q_{n}\right) q_{n}^{-s}$, where $1 \leqslant q_{1}<q_{2}<\cdots$ and $q_{n} \rightarrow \infty$ as $n \rightarrow \infty$, and where the $G\left(q_{n}\right)$ are real coefficients with the property that there exist constants $C \neq 0$ and $\alpha, \beta$ with $0 \leqslant \beta<\alpha$ such that

$$
N_{G}(x)={ }_{\text {def. }} \sum_{4_{n} \leqslant x} G\left(q_{n}\right)=C x^{\alpha}+O\left(x^{\beta}\right) \text { as } x \rightarrow \infty .
$$

Then the abscissa of convergence of $\zeta_{G}(s)$ is $\alpha$, and $\zeta_{G}(s)$ can be extended to an analytic function of $s$ for all complex $s$ with $\operatorname{Re} s>\beta, s \neq \alpha$, in such a way that, about $s=\alpha, \zeta_{G}(s)$ has a Laurent expansion of the form

$$
\zeta_{G}(s)=\frac{\alpha C}{s-\alpha}+\sum_{i=0}^{\infty} \frac{(-1)^{i}}{i !} \gamma_{i}(G)(s-\alpha)^{i}
$$

where

$$
\gamma_{i}(G)=\sum_{q_{n}=x} G\left(q_{n}\right) q_{n}^{-\alpha} \log ^{i} q_{n}-\frac{\alpha C}{i+1} \log ^{i+1} x+O\left(x^{\beta-\alpha} \log ^{i} x\right)
$$

Proof. Let $F(0)=0$, and $F(t)=N_{G}\left(e^{t}\right)$ for $t>0$. Then $F(t)$ is a step function with jumps $G\left(q_{n}\right)$ at the points $t=\log q_{n}$. Hence, for a continuous function $\psi(t)$,

$$
\sum_{q_{n}<x} G\left(q_{n}\right) \psi\left(\log q_{n}\right)=\int_{0}^{\log x} \psi(t) d F(t) .
$$

Then the asymptotic hypothesis on $N_{G}(x)$ implies that $\dot{\zeta_{G}}(s)=\int_{0}^{\infty} e^{-s t} d F(t)$ for $\operatorname{Re} s>$ 
$\alpha$, and so the corollary follows immediately from Theorem 1.1, the present asymptotic formula for $\gamma_{i}(G)=\gamma_{i}(F)$ following from that of Theorem 1.1 upon substitution of $t^{i} e^{-\alpha t}$ for $\psi(t)$ in the equation above.

The main theorem of Briggs and Buschman (5) follows from Corollary 1.2, in the special case when $q_{n}=n$ for $n=1,2, \ldots$, so that $\zeta_{G}(s)$ becomes an ordinary Dirichlet series; Briggs and Buschman did not provide an error estimate in their limit relation for $\gamma_{i}(G)$.

\section{Examples and applications}

Consider the Hurwitz zeta function $\zeta(s, a)=\sum_{n=0}^{\infty}(n+a)^{-s}, 0<a \leqslant 1$. Here the function $N_{G}(x)$ of Corollary 1.2 is asymptotically $x+O(1)$ as $x \rightarrow \infty$. Hence application of Corollary 1.2 yields Theorem 1 of Berndt (2), together with an additional error estimate in the limit relation for the generalised Euler constants.

Another example of interest is the zeta function

$$
\zeta_{Q}(s)=\sum_{m, n}^{\prime} Q(m, n)^{-s}
$$

of a positive definite real quadratic form $Q(x, y)=a x^{2}+b x y+c y^{2}$ of negative discriminant $b^{2}-4 a c=-\Delta$. A result of Gauss (see for example Landau (10), page 166) implies that the function corresponding to $N_{G}(x)$ in Corollary 1.2 is asymptotically $C x+O\left(x^{1 / 2}\right)$, where $C=2 \pi / \sqrt{ } \Delta$. Thus Corollary 1.2 may be applied to this function. The resulting conclusions for the constant $\gamma_{0}(Q)$ and their relation with the Kronecker limit formula (see also Siegel (14), pages 5-6) were discussed by Landau (10), page 168. Those for the higher constants $\gamma_{i}(Q)$, though certainly not comparable in depth with the Kronecker formula, seem at least worth mentioning, since they do not seem to appear in the literature.

A quite comprehensive class of examples is covered by the cases in which the function $\zeta_{G}(s)$ of Corollary 1.2 is the zeta function of an arithmetical semigroup satisfying Axiom A, or of a class in an arithmetical formation satisfying Axiom $A^{*}$, as defined and discussed by the author ((8), Parts II, V, and (9), Chapter 9). Here, the first constant $\gamma_{0}(G)$ was discussed previously by this author (loc. cit.), but rather than elaborate upon details concerning the higher constants $\gamma_{i}(G)$ in full generality we shall now restrict attention to a selection of specific functions of interest:

First consider the Dedekind zeta function $\zeta_{K}(s)=\sum_{n=1}^{\infty} K(n) n^{-s}$ of an algebraic number field $K$, where $K(n)$ denotes the number of integral ideals of norm $n$ in $K$. By a theorem of Weber (see Landau (12), page 135),

$$
\sum_{n \leq x} K(n)=A_{K} x+O\left(x^{n}\right) \text { as } x \rightarrow \infty,
$$

where $A_{K}$ and $\eta$ are explicitly definable positive constants depending on $K$. Landau $(10,11)$ gave limit relations with error estimates for the resulting Euler constants $\gamma_{i}(K)$ of $K$, but without reference to the interpretation of $\gamma_{i}(K)(i \geqslant 1)$ in the Laurent expansion of $\zeta_{K}(s)$ about $s=1$.

A different type of example arises from consideration of the arithmetical function 
$a(n)$ defined by the total number of non-isomorphic abelian groups of order $n$ $(n=1,2, \ldots)$. In the terminology used in $(8,9)$, the corresponding Dirichlet series $\zeta_{a}(s)=\sum_{n=1}^{\infty} a(n) n^{-s}$ is the zeta function of the arithmetical semigroup $\mathscr{A}$ of all isomorphism classes of finite abelian groups.

Theorem 2.1. The zeta function $\zeta_{9}(s)$ possesses generalised Euler constants $\gamma_{i}(\mathcal{U})$ satisfying the relations

$$
\begin{aligned}
\gamma_{i}(\mathfrak{A}) & =\frac{(-1)^{i}}{i+1} Z_{\mathfrak{a}}^{(i+1)}(1)+\sum_{j=0}^{i}(-1)^{i+j}\left(\begin{array}{l}
i \\
j
\end{array}\right) \gamma_{j} Z_{\mathfrak{a}}^{(i-j)}(1) \\
& =\sum_{n=1}^{N} a(n) \frac{\log ^{i} n}{n}-\frac{A}{i+1} \log ^{i+1} N+O\left(N^{-1 / 2} \log ^{i} N\right)
\end{aligned}
$$

where $Z_{q}(s)=\Pi_{r=2}^{\infty} \zeta(r s), \zeta(s)$ is the Riemann zeta function, the $\gamma_{j}$ are the generalised Euler constants of $\zeta(s)$, and $A=Z_{\mathrm{q}}(1)=2 \cdot 294 \ldots$

Proof. For the case $i=0$ of this theorem, see also Cohen (7) and the author $(8)$, Part II. For the proof, we note that theorems of Erdös and Szekeres and of Kendall and Rankin (see for example (9), Chapter 5) imply that $\zeta_{9}(s)=\zeta(s) Z_{g}(s)$ for $\operatorname{Re} s>0$, and that $\Sigma_{n<x} a(n)=A x+O\left(x^{1 / 2}\right)$ as $x \rightarrow \infty$. Hence Corollary 1.2 is applicable, and yields the limit relation for $\gamma_{i}(\mathfrak{U})$. The other relation for $\gamma_{i}(\mathfrak{U})$ may then be obtained by calculating the Laurent expansion about $s=1$ of

$$
\zeta(s) Z_{\mathrm{g}}(s)=\left\{\frac{1}{s-1}+\sum_{i=0}^{\infty} \frac{(-1)^{i}}{i !} \gamma_{i}(s-1)^{i}\right\} Z_{\mathrm{g}}(s)
$$

Another example arising from abstract algebra may be obtained by considering the arithmetical function $S(n)$, which is defined by the total number of non-isomorphic semisimple finite rings of order $n(n=1,2,3, \ldots)$. In the terminology used in $(8,9)$, $\zeta_{\varsigma}(s)=\sum_{n=1}^{\infty} S(n) n^{-s}$ is the zeta function of the arithmetical semigroup $S$ of all isomorphism classes of semisimple finite rings.

Theorem 2.2. The zeta function $\zeta_{\circlearrowleft}(s)$ possesses generalised Euler constants $\gamma_{i}\left(\Phi_{)}\right)$ satisfying the relations

$$
\begin{aligned}
\gamma_{i}(\Im) & =\frac{(-1)^{i}}{i+1} Z_{\Im}^{(i+1)}(1)+\sum_{j=0}^{i}(-1)^{i+i}\left(\begin{array}{l}
i \\
j
\end{array}\right) \gamma_{i} Z_{\Im}^{(i-i)}(1) \\
& =\sum_{n=1}^{N} S(n) \frac{\log ^{i} n}{n}-\frac{B}{i+1} \log ^{i+1} N+O\left(N^{-1 / 2} \log ^{i} N\right)
\end{aligned}
$$

where $Z_{\Im}(s)=\prod_{m>1} \zeta\left(\mathrm{rm}^{2} s\right)$, and $B=Z_{\Im}(1)=2 \cdot 499 \ldots$

Proof. This theorem follows in the same way as Theorem 2.1, upon applying Corollary 1.2 to a result of the author (8), Part I, which states that $\Sigma_{n<x} S(n)=$ $B x+O\left(x^{1 / 2}\right)$ as $x \rightarrow \infty$, and $\zeta_{\varsigma}(s)=\zeta(s) Z_{\Im}(s)$ for $\operatorname{Re} s>\frac{1}{2}$.

Without going into further details, it may be remarked that generalisations of Theorems 2.1 and 2.2, concerning molecules of finite cardinal or semisimple finite 
algebras over the ring of all algebraic integers in an algebraic number field $K$, can be derived similarly with the aid of theorems of the author (8), Part III. In these generalisations, the rôle of the constants $\gamma_{i}$ is taken by the constants $\gamma_{i}(K)$ of $K$ that were mentioned above.

\section{Constants of arithmetical progressions}

Consider the arithmetical progression $H=H(r, k)$ consisting of positive integers $r$, $r+k, r+2 k, \ldots(r \leqslant k)$. For the corresponding Dirichlet series $\zeta_{H}(s)=\Sigma_{n \in H} n^{-s}$, one has $\Sigma_{n \leqslant x, n \in H} 1=(1 / k) x+O(1)$ as $x \rightarrow \infty$. Therefore Corollary 1.2 implies that there exists a sequence of generalised Euler constants $\gamma_{i}(r, k)$ corresponding to $H$. The first of these constants $\gamma_{0}(r, k)$ was studied previously by Briggs (4) and Lehmer (13), and as supplements to the results of these authors we mention here a few corresponding ones about the higher constants.

Firstly, it is clear that

$$
\sum_{r=1}^{k} \gamma_{i}(r, k)=\gamma_{i}(1,1)=\gamma_{i}
$$

where $\gamma_{i}$ corresponds to $\zeta(s)$ as before. Further, one has:

Proposition 3.1. For $r=r^{\prime} d, k=k^{\prime} d$,

$$
\gamma_{i}(r, k)=\frac{1}{d} \sum_{i=0}^{i}\left(\begin{array}{l}
i \\
j
\end{array}\right) \gamma_{i-j}\left(r^{\prime}, k^{\prime}\right) \log ^{i} d-\frac{\log ^{i+1} d}{k(i+1)}
$$

in particular,

$$
\gamma_{i}(k, k)=\frac{1}{k}\left\{\sum_{j=0}^{i}\left(\begin{array}{l}
i \\
j
\end{array}\right) \gamma_{i-j} \log ^{i} k-\frac{\log ^{i+1} k}{i+1}\right\}
$$

Proof. Let $H^{\prime}$ denote the progression $r^{\prime}, r^{\prime}+k^{\prime}, r^{\prime}+2 k^{\prime}, \ldots$ Then, by Corollary 1.2 ,

$$
\begin{aligned}
\gamma_{i}(r, k) & =\lim _{x \rightarrow \infty}\left\{\sum_{n \leqslant x, n \in H} \frac{\log ^{i} n}{n}-\frac{\log ^{i+1} x}{k(i+1)}\right\} \\
& =\lim _{x \rightarrow \infty} \frac{1}{d}\left\{\sum_{m d \in x, m \in H^{\prime}} \frac{1}{m} \sum_{j=0}^{i}\left(\begin{array}{l}
i \\
j
\end{array}\right) \log ^{i} d \cdot \log ^{i-j} m-\frac{\log ^{i+1} x}{k^{\prime}(i+1)}\right\} .
\end{aligned}
$$

Hence the proposition follows from the relations:

$$
\begin{aligned}
\frac{\log ^{i+1} x}{i+1} & =\frac{1}{i+1} \sum_{j=0}^{i+1}\left(\begin{array}{c}
i+1 \\
j
\end{array}\right) \log ^{j} d \cdot \log ^{i+1-i}(x / d) \\
& =\frac{\log ^{i+1} d}{i+1}+\sum_{i=0}^{i}\left(\begin{array}{l}
i \\
j
\end{array}\right) \log ^{i} d \cdot \frac{\log ^{i-j+1}(x / d)}{i-j+1}
\end{aligned}
$$

For the case $i=0$ of the above and following conclusions, see Briggs (4) and Lehmer (13).

Proposition 3.2. Let $g(n)$ denote an arithmetical function which is periodic in $n$, 
of period $k>0$. Then the series $\sum_{n=1}^{\infty} g(n)\left(\log ^{i} n / n\right)$ is convergent, and has the sum $\sum_{r=1}^{k} g(r) \gamma_{i}(r, k)$, if and only if $\sum_{r=1}^{k} g(r)=0$.

Proof. This is a direct consequence of the identity:

$$
\begin{aligned}
\sum_{n<x} g(n) \frac{\log ^{i} n}{n}= & \sum_{r=1}^{k} g(r)\left\{\sum_{n<x, n=r(\bmod k)} \frac{\log ^{i} n}{n}-\frac{\log ^{i+1} x}{k(i+1)}\right\} \\
& +\frac{\log ^{i+1} x}{k(i+1)} \sum_{r=1}^{k} g(r) .
\end{aligned}
$$

Corollary 3.3. For any non-principal Dirchlet character $\chi$ modulo $k>1$, the value of the $i$-th derivative of $L(s, \chi)=\sum_{n=1}^{\infty} \chi(n) n^{-s}$ at $s=1$ is

$$
L^{(i)}(1, \chi)=(-1)^{i} \sum_{r=1}^{k} \chi(r) \gamma_{i}(r, k) \text {. }
$$

For the case $i=0$ of this corollary, see Berndt (3) as well as Lehmer (13).

Lastly, consider the constant

$$
\gamma_{i}(k)=\sum_{i \leqslant t \leqslant k, t(k)=1} \gamma_{i}(t, k)
$$

this may be regarded as the $i$-th generalised Euler constant of the multiplicative semigroup of all positive integers coprime to the given integer $k>0$. This semigroup has the zeta function

$$
\begin{aligned}
\zeta(s ; k) & =\sum_{n \geqslant 1 .\{n, k)=1} n^{-s}=\zeta(s) \prod_{\text {prime } p \mid k}\left(1-p^{-s}\right) \\
& =\zeta(s) \prod_{p \mid t}\left\{1-\frac{1}{p} \sum_{j=0}^{\infty} \frac{(-1)^{j}}{j !} \log ^{j} p \cdot(s-1)^{j}\right\} .
\end{aligned}
$$

This last equation implies:

Proposition 3.4. The constant $\gamma_{i}(k)$ satisfies:

$$
\frac{(-1)^{i}}{i !} \gamma_{i}(k)=a_{i+1}+\sum_{j=0}^{i} \frac{(-1)^{i}}{j !} \gamma_{j} a_{i-j}
$$

where, for $c_{0}(p)=1-p^{-1}$ and $c_{i}(p)=(-1)^{i+1} p^{-1} \log ^{i} p(i \geqslant 1)$,

$$
a_{j}=\sum\left\{\prod_{\text {prime } p \mid k} c_{i_{p}}(p): \sum_{p} i_{p}=j\right\} \text {. }
$$

\section{REFERENCES}

(1) P. T. Bateman and H. G. Diamond, Asymptotic distribution of Beurling's generalized prime numbers, Studies in Number Theory, MAA Studies in Math., Vol. 6 (Prentice-Hall, 1969).

(2) B. C. BERndT, On the Hurwitz zeta function, Rocky Mountain J. Math. 2 (1972), 151-157. 
(3) B. C. BERNDT, Character analogues of the Poisson and Euler-MacLaurin summation formulas with applications, J. Number Theory 7 (1975), 413-445.

(4) W. E. BRIGgs, The irrationality of $\gamma$ or of sets of similar constants, K. Norske Vid. Selsk. Forh. (Trondheim) 34 (1961), 25-28.

(5) W. E. BRIGGS \& R. G. BUSCHMAN, The power series coefficients of functions defined by Dirichlet series, Illinois J. Math. 5 (1961), 43-44.

(6) W. E. Briggs \& S. Chowla, The power series coefficients of $\zeta(s)$, Amer. Math. Monthly 62 (1955), 323-325.

(7) E. COHEN, On the average number of direct factors of a finite abelian group, Acta Arith. 6 (1960), 159-173.

(8) J. KNOPFMACHER, Arithmetical properties of finite rings and algebras, and analytic number theory, I-V, J. Reine Angew. Math. 252 (1972), 16-43, 254 (1972), 74-99, 259 (1973), 157-170, 270 (1974), 97-114, 271 (1974), 95-121. 1975).

(9) J. KNOPFMACHER, Abstract Analytic Number Theory (North-Holland Publ. Co.,

(10) E. LANDAU, Über die zu einem algebraischen Zahlkörper gehörige Zetafunktion ..., J. Reine Angew. Math. 125 (1903), 64-188. $1-21$.

(11) E. Landau, Über eine idealtheoretische Funktion. Trans. Amer. Math. Soc. 13 (1912),

(12) E. LANDAU, Einführung in die elementare und analytische Theorie der algebraischen Zahlen und der Ideale (Chelsea Publ. Co., 1949 reprint).

(13) D. H. LeHMER, Euler constants for arithmetical progressions, Acta Arith. 27 (1975), $125-142$.

(14) C. L. SiEgEL, Lectures on Advanced Analytic Number Theory (Tata Inst. of Fund. Research, 1961).

(15) S. C. VAN VEen, Math. Reviews 29 (1965) \#2232.

(16) D. V. WIDDER, The Laplace Transform (Princeton Univ. Press, 1941).

\section{UNIVERSITY OF WITWATERSRAND,}

JOHANNESBURG

SOUTH AFRICA 